\title{
Sensitivity analysis for the EEG forward problem
}

\author{
Maria Inés Troparevsky ${ }^{*}$, Diana Rubio ${ }^{2}$ and Nicolas Saintier ${ }^{3}$ \\ Departamento de Matemática, Facultad de Ingeniería, Universidad de Buenos Aires, Buenos Aires, Argentina \\ 2 Centro de Matemática Aplicada, Escuela de Ciencia y Tecnologia, Universidad Nacional General San Martín, Buenos Aires, Argentina \\ 3 Instituto de Ciencias, Universidad Nacional General Sarmiento, Buenos Aires, Argentina
}

\section{Edited by:}

Klaus R. Pawelzik, University of

Bremen, Germany

\section{Reviewed by:}

H. T. Banks, North Carolina State

University, USA

Franz Kappel, University of Graz,

Austria

\section{*Correspondence:}

Maria Inés Troparevsky, Departamento de Matemática, Facultad de Ingenieria, Universidad de Buenos Aires, Av.

Paseo Colón 850, C1063ACV Buenos

Aires, Argentina.

e-mail:mitropa@fi.uba.ar

\begin{abstract}
Sensitivity analysis can provide useful information when one is interested in identifying the parameter $\theta$ of a system since it measures the variations of the output $u$ when $\theta$ changes. In the literature two different sensitivity functions are frequently used: the traditional sensitivity functions (TSF) and the generalized sensitivity functions (GSF). They can help to determine the time instants where the output of a dynamical system has more information about the value of its parameters in order to carry on an estimation process. Both functions were considered by some authors who compared their results for different dynamical systems (see Banks and Bihari, 2001; Kappel and Batzel, 2006; Banks et al., 2008). In this work we apply theTSF and the GSF to analyze the sensitivity of the 3D Poisson-type equation with interfaces of the forward problem of electroencephalography. In a simple model where we consider the head as a volume consisting of nested homogeneous sets, we establish the differential equations that correspond toTSF with respect to the value of the conductivity of the different tissues and deduce the corresponding integral equations. Afterward we compute the GSF for the same model. We perform some numerical experiments for both types of sensitivity functions and compare the results.
\end{abstract}

Keywords: forward problem of electroencephalography, sensitivity, traditional sensitivity, generalized sensitivity

\section{INTRODUCTION}

The electric process underlying the generation of the electroencephalography (EEG) signals can be modeled as a set of current sources within the brain. Considering the velocity of propagation of the electric waves in the brain, the static approximation of Maxwell's equations can be used to describe this process (see Hamalainen et al., 1993). The resulting model is a 3D Poisson-type equation with interfaces that relates the electric potential $u$ in the head $G$ with the impressed current $J_{i}$ often represented by an electric dipole, i.e., $J_{i}(x)=M \delta(x-q)$, where $\delta$ is the Dirac distribution, $q$ is a fixed point in the brain which represents the dipole location, and $M$ is the dipole moment. The parameters of the model are the conductivity of the different tissues, $\sigma(x), x \in G$, the diameters of the different compartments of the head, and the moment and location of the dipole: $M \in R^{3}, q \in G$ respectively. The forward problem (FP) of EEG consists in finding the electric potential $u$ in the head for a given current source $J_{i}$. Conversely the inverse problem (IP) of EEG consists in finding the location $q$ that produces a given scalp data $u$. An accurate solution to the IP could provide useful information to treat neurological diseases such as epilepsy. In order to solve the IP we need first to solve the FP. In this context it is important to analyze the sensitivity of the solutions to the FP with respect to the different parameters of the model since this information could help us to choose the points on the scalp where the data are to be collected.

In the literature two different sensitivity functions are frequently used: the traditional sensitivity functions (TSF) defined as $\partial \mathrm{u} / \partial \theta$ that measures the variations of the output $u$ when the parameter $\theta$ changes and the generalized sensitivity functions (GSF) that determine at which time instants the output of a dynamical system has more information about the value of its parameters in order to carry on an estimation process.
In this work we consider the head as a multi compartment nested set and assume homogeneity and isotropy, hence the conductivity function $\sigma(x)$ that contains the value of the conductivity of the different tissues is approximated by a positive piecewise constant function. We analyze the sensitivity of the solution $u$ to the FP with respect to the conductivity $\sigma(x)$ by means of the TSF and the GSF and compare the results.

The paper is organized as follows. In Section "The Mathematical Model" we present the mathematical model. The TSF are introduced in Section "Materials and Methods." Differential and integral equations are stated. In Section "Results," we present the GSF. Based on Rubio et al. (2009) we calculate the GSF of $u$ with respect to $\sigma(x)$. We performed numerical experiments in a simple head model and present some conclusions.

\section{THE MATHEMATICAL MODEL}

The electrical activity of the brain consists of currents generated by biochemical sources at cellular level. The electric and magnetic fields that they produce can be estimated by means of Maxwell's equations (see Sarvas, 1987; Hamalainen et al., 1993). Based on the properties of the tissues involved, the velocity of propagation of the electromagnetic waves caused by potential changes within the brain is such that the effect may be detected simultaneously at any point in the brain or in the surrounding tissues. This fact justifies the use of a static approximation of Maxwell's equations. This approximation uncouples the equations for the magnetic and electric fields leading to the following second order partial differential equation (PDE)

$\nabla \cdot(\sigma(x) \nabla u(x))=\nabla \cdot J_{i}(x) \quad x \in G$, 
that relates the measured electric potential $u$ and the impressed current $J_{i}$.

The volume $G$ (head model) consists of nested homogeneous sets, one surrounded by the next one where the conductivity values are given.

We consider $G=\bigcup_{j=1}^{3} G_{j}$, denoted from the inner one to the outer one by: $G_{1}$, the brain, $G_{2}$, the skull, and $G_{3}$, the scalp. We denote $\Gamma=\partial G$ the external surface of $G$ and by $S_{1}=\partial G_{1}, S_{2}=\partial G_{2}-\partial G_{1}$ the surfaces between $G_{1}$ and $G_{2}$ and $G_{2}$ and $G_{3}$ respectively.

The positive, discontinuous and piecewise constant function $\sigma(x)$ that contains the conductivity values of the different tissues at each point is:

$\sigma(x)=\left\{\begin{array}{ccc}\sigma_{1} & x & \in G_{1} \\ \sigma_{2} & x & \in G_{2} \\ \sigma_{3} & x & \in G_{3} \\ 0 & x & \notin G\end{array}\right.$.

Assuming that the potential and its normal derivative times the conductivity function are continuous across the transition surfaces, the resulting boundary value problem is:

$\nabla \cdot(\sigma(x) \nabla u(x))=F(x) \quad x \in G$

$\frac{\partial u(x)}{\partial v}=0, \quad x \in \partial G$

subject to:

$\left.[u]\right|_{S_{i}^{-}}=\left.0 \quad\left[\sigma(x) \frac{\partial u}{\partial v}\right]\right|_{S_{i}^{-}}=0 \quad i=1,2$.

where $[\cdot]$ denotes the difference between the values of the functions inside the brackets through the indicated surface, $v$ is the external normal vector and $F(x)=\nabla \cdot J_{i}(x)$.

If $F$ is regular enough, there exist solutions (in a weak sense) to (3) provided that $F$ verifies $\int_{G} F(x) d x=0$. They are unique up to a constant (see Troparevsky and Rubio, 2003).

When $J_{\mathrm{i}}$ is an electric dipole, existence and uniqueness of solutions were studied in El Badia (2000).

\section{MATERIALS AND METHODS}

\section{TRADITIONAL SENSITIVITY FUNCTIONS}

The TSF is a common tool used to measure the variation of the output $u$ of a system with respect to changes in its parameter $\theta=\left(\theta_{1}\right.$, $\left.\theta_{2}, \ldots, \theta_{p}\right)$. Assuming smoothness of $u$, they are defined as the partial derivative of $u$ with respect to each $\theta_{i}$, namely:

$s_{i}(x)=\frac{\partial u}{\partial \theta_{i}}(x), \quad i=1, \ldots, p$

The sensitivity functions are related to $u$ by the Taylor approximation of first order. In the case of the system modeled by equations (1)-(5), considering $u$ as a function of $x \in G$ and the vector of parameters $\theta=\left(\sigma_{1}, \sigma_{3} \sigma_{3}\right)$, i.e., $u=u\left(x, \sigma_{1}, \sigma_{1}, \sigma_{3} \sigma_{3}\right)$ and fixing $x, \sigma_{2}, \sigma_{3}$, we have:

$$
u\left(x, \sigma_{1}+\Delta, \sigma_{2,} \sigma_{3}\right) \cong u\left(x, \sigma_{1}, \sigma_{2}, \sigma_{3}\right)+s_{1}\left(x, \sigma_{1}, \sigma_{2}, \sigma_{3}\right) \Delta
$$

These functions $s_{i}(x)$ give local information and are used to determine the parameter to which the model is more sensitive. Note that the value of $s_{i}(x)$ depends on the dimensions and the units of the parameter $\theta_{i}$, then, in order to compare the sensitivity with respect to different parameters "normalized sensitivities" can be introduced, where the quotient between the relative errors of the output (numerator) and the relative error of the parameter (denominator) is considered.

In the next subsection we derive the differential sensitivity equations (see Stanley, 1999; Burns et al., 2005) for the interface problem (3)-(5) introduced in Section "The Mathematical Model."

\section{Differential equations for the TSF}

We consider three parameters in our model: the conductivity values for each sub domain $G_{i}$, i.e., $\sigma_{1}, \sigma_{2}, \sigma_{3}$ Thus the vector of parameters is $\theta=\left(\theta_{1}, \theta_{2}, \theta_{3}\right)=\left(\sigma_{1}, \sigma_{2}, \sigma_{3}\right)$, and the sensitivity functions to be considered are:

$s_{1}(x)=\frac{\partial u}{\partial \sigma_{1}}(x), \quad s_{2}(x)=\frac{\partial u}{\partial \sigma_{2}}(x), \quad s_{3}(x)=\frac{\partial u}{\partial \sigma_{3}}(x)$

These functions satisfy the following state equations that can be derived from (3) by formal differentiation (see Stanley, 1999):

$$
\begin{array}{rlrlr}
\Delta u+\sigma_{1} \Delta s_{1} & =0 & x \in G_{1} & \sigma_{1} \Delta s_{2}=0 & x \in G_{1} \\
\sigma_{2} \Delta s_{1}=0 & x \in G_{2} & \Delta u+\sigma_{2} \Delta s_{2}=0 & x \in G_{2} \\
\sigma_{3} \Delta s_{1}=0 & x \in G_{3} & \sigma_{3} \Delta s_{3}=0 & x \in G_{3} \\
\sigma_{1} \Delta s_{3}=0 & x \in G_{1} & & \\
\sigma_{2} \Delta s_{3}=0 & x \in G_{2} & & \\
\Delta u+\sigma_{3} \Delta s_{3} & =0 & x \in G_{3} & &
\end{array}
$$

The boundary condition for each $s_{i}$ can be derived from (4) observing that the normal $v$ on the external surface $\Gamma=\partial G$ does not depend on the $\sigma_{i}, i=1,2,3$.

$\left.\frac{\partial s_{i}}{\partial v}\right|_{\partial G}=0 \quad i=1,2,3$.

The equations (5) of continuity of $u$ at the transition surfaces lead to:

$\left.s_{i}\right|_{S_{j}^{-}}-\left.s_{i}\right|_{S_{j}^{+}}=0, \quad i=1,2,3 . \quad j=1,2$.

and,

$\left\{\begin{array}{l}\left.\sigma_{1} \frac{\partial s_{1}}{\partial v}\right|_{S_{1}^{-}}-\left.\sigma_{2} \frac{\partial s_{1}}{\partial v}\right|_{S_{1}^{+}}=-\left.\frac{\partial u}{\partial v}\right|_{S_{1}^{-}} \\ \left.\sigma_{2} \frac{\partial s_{1}}{\partial v}\right|_{S_{2}^{-}}-\left.\sigma_{3} \frac{\partial s_{1}}{\partial v}\right|_{S_{2}^{+}}=0\end{array}\right.$

$\left\{\begin{array}{l}\left.\sigma_{1} \frac{\partial s_{2}}{\partial v}\right|_{S_{1}^{-}}-\left.\sigma_{2} \frac{\partial s_{2}}{\partial v}\right|_{S_{1}^{+}}=-\left.\frac{\partial u}{\partial v}\right|_{S_{1}^{+}} \\ \left.\sigma_{2} \frac{\partial s_{2}}{\partial v}\right|_{S_{2}^{-}}-\left.\sigma_{3} \frac{\partial s_{2}}{\partial v}\right|_{S_{2}^{+}}=-\left.\frac{\partial u}{\partial v}\right|_{S_{2}^{-}}\end{array}\right.$

$\left\{\begin{array}{l}\left.\sigma_{1} \frac{\partial s_{3}}{\partial v}\right|_{S_{1}^{-}}-\left.\sigma_{2} \frac{\partial s_{3}}{\partial v}\right|_{S_{1}^{+}}=0 \\ \left.\sigma_{2} \frac{\partial s_{3}}{\partial v}\right|_{S_{2}^{-}}-\left.\sigma_{3} \frac{\partial s_{3}}{\partial v}\right|_{S_{2}^{+}}=+\left.\frac{\partial u}{\partial v}\right|_{S_{2}^{+}}\end{array}\right.$ 
Note that in order to solve the PDE systems that correspond to the sensitivity functions $s_{i}(x)$ one needs the values of $u$ and $\partial u / \partial v$ on the transition surfaces and the value of the Laplacian $\Delta u$ on $G$.

As for the equation (3), the sensitivity equations may only have solution in a weak sense.

\section{Integral equations}

Integral equations for the sensitivity functions can also be stated. They are useful to perform numerical approximations. We present the ones corresponding to $s_{1}(x)$.

Recall that $s_{1}$ satisfies:

$\Delta u+\sigma_{1} \Delta s_{1}=0 \quad x \in G_{1}$

$$
\begin{array}{ll}
\sigma_{2} \Delta s_{1}=0 & x \in G_{2} \\
\sigma_{3} \Delta s_{1}=0 & x \in G_{3}
\end{array}
$$

with boundary condition:

$$
\left.\frac{\partial s_{i}}{\partial v}\right|_{\partial G}=0 \quad i=1,2,3 .
$$

and interface conditions:

$$
\begin{gathered}
\left.s_{1}\right|_{S_{j}^{-}}=\left.s_{1}\right|_{S_{j}^{+}}, \quad j=1,2,\left.\quad \sigma_{1} \frac{\partial s_{1}}{\partial v}\right|_{S_{1}^{-}}-\left.\sigma_{2} \frac{\partial s_{1}}{\partial v}\right|_{S_{1}^{+}}=-\left.\frac{\partial u}{\partial v}\right|_{s_{1}^{-}}, \\
\left.\sigma_{2} \frac{\partial s_{1}}{\partial v}\right|_{S_{2}^{-}}-\left.\sigma_{3} \frac{\partial s_{1}}{\partial v}\right|_{S_{2}^{+}}=0
\end{gathered}
$$

Applying green integral identity:

$$
\int_{G_{i}} s_{1} \Delta \Phi-\Phi \Delta s_{1} d x=\int_{\partial G_{i}} s_{1} \nabla \Phi-\Phi \nabla s_{1} d S
$$

in each $G_{i}, i=1,2$ and choosing $\Phi$ such that $\Delta \Phi(x, y)=-4 \pi \delta(x-y)$, i.e., $\Phi(x, y)=1 /|x-y|$ we arrive to:

$$
\begin{aligned}
& \sum_{i=1}^{3}\left[\sigma_{i} s_{1}(x)(-4 \pi) \chi_{G_{i}}(x)-\sigma_{i} \int_{G_{i}} \Phi \Delta s_{1} d y\right] \\
& =\sigma_{1} \int_{\partial G_{i}^{-}} s_{1}(y) \frac{\partial \Phi}{\partial v}(x, y) d S y-\sigma_{i} \int_{\partial G_{i}^{-}} \Phi(x, y) \frac{\partial s_{1}}{\partial v}(y) d S y .
\end{aligned}
$$

Combining the former equation with (7), the boundary condition (9) and the continuity conditions at the transition surfaces (10) we obtain:

$$
\begin{aligned}
-4 \pi s_{1}(x) \chi G_{1}(x) \sigma_{1}+ & \int_{G_{1}} \Phi(x, y) \Delta u(y) d y-4 \pi s_{1}(x) \chi G_{2}(x) \sigma_{2} \\
-4 \pi s_{1}(x) \chi G_{3}(x)= & \left(\sigma_{1}-\sigma_{2}\right) \int_{S_{1}^{+}} s_{1} \frac{\partial \Phi}{\partial v} d S y \\
& +\int_{S_{1}^{+}} \frac{\partial u}{\partial v} \Phi d S y+\left(\sigma_{2}-\sigma_{3}\right) \int_{S_{2}^{+}} \frac{\partial \Phi}{\partial v} s_{1} d S y \\
& +\left(\sigma_{3}-\sigma_{4}\right) \int_{\partial G^{+}} s_{1}(y) \frac{\partial \Phi}{\partial v} d S y .
\end{aligned}
$$

If we take $x \in G_{1}$ in (13), since $\sigma_{1} \Delta u=F$ in $G_{1}$ we obtain:

$$
\begin{aligned}
\sigma_{1} s_{1}(x)= & -\frac{1}{4 \pi \sigma_{1}} \int_{G_{1}} F(y) \frac{1}{|x-y|} d y \\
& -\frac{1}{4 \pi} \int_{S_{1}^{+}} \frac{\partial u}{\partial v} \Phi+\frac{\sigma_{2}-\sigma_{1}}{4 \pi} \int_{S_{1}^{+}} s_{1} \frac{\partial \Phi}{\partial v} \\
& +\frac{\sigma_{3}-\sigma_{2}}{4 \pi} \int_{S_{2}^{+}} s_{1} \frac{\partial \Phi}{\partial v} \Phi d S y+\frac{\sigma_{4}-\sigma_{3}}{4 \pi} \int_{\Gamma^{+}} s_{1}(y) \frac{\partial \Phi}{\partial v} d S y .
\end{aligned}
$$

Similarly we can choose $x \in G_{2}$ or $x \in G_{3}$ to obtain the corresponding integral equations.

Remark Note that only the values of $s_{1}$ on the transition surfaces appear in the right hand side of (14).

Letting $x \rightarrow \bar{x} \in S_{1}$ in (14), and recalling that:

$$
\begin{aligned}
\lim _{x \in \Omega \rightarrow x \in \partial \Omega} \int_{\partial \Omega^{-}} f(y) \frac{x-y}{|x-y|^{3}} d S y= & -2 \pi f(\bar{x}) \\
& +\int_{\partial \Omega-\{\bar{x}\}} f(\bar{x}) \frac{x-y}{|x-y|^{3}} d S y,
\end{aligned}
$$

we can obtain an equation involving only values of $s_{1}$ on the transition surfaces.

\section{GENERALIZED SENSITIVITY FUNCTION}

The GSF was introduced by Thomaseth and Cobelli (1999) to analyze qualitatively where the information about model parameters is concentrated during identification experiments. It was meant to understand how the estimation is related to observed system outputs, taking into account correlations between the model parameters. We recall the definition and some properties of the GSF, for details we refer to Thomaseth and Cobelli (1999).

Consider a non-linear parametric dynamical system:

$\dot{u}(t)=f(t, u, \theta)$

$u\left(t_{0}\right)=u_{0}$

with a set of discrete time observations at $t_{1} \ldots t_{\mathrm{n}}$ :

$y_{j}(\theta)=h\left(t_{j}, \theta\right)+e_{j}, \quad j=1, \ldots, n$

where $u(t), f(t, u, \theta), \in R^{N}, \theta \in R^{p}, h(t, \theta) \in R$, and $e_{i}, \in R$ is the measurement noise assumed to be independently and identically distributed with zero mean and known variance $v_{j}^{2}$. It is assumed that there exists a true $\theta_{0}$ such that the observed data $y_{j}$ correspond to that parameter value.

Let us consider the minimization of the weighted residual sum of squares:

$J\left(y, \theta_{0}\right)=\sum_{j=1}^{n} \frac{\left(y_{j}-h\left(t_{j}, \theta_{0}\right)\right)^{2}}{v_{j}^{2}}$.

A value $\hat{\theta}$ where the minimum is attained is an estimator of $\theta_{0}$. 
The GSF at $t_{k}$ are defined as:

$$
\begin{aligned}
g s\left(t_{k}\right) & =\sum_{j=1}^{k} \frac{1}{\sigma^{2}\left(t_{j}\right)}\left[F^{-1} \times \nabla_{\theta} h\left(t_{j}, \theta_{0}\right)\right] \cdot \nabla_{\theta} h\left(t_{j}, \theta_{0}\right), \\
k & =1, \ldots, n, \quad l=1, \ldots, p
\end{aligned}
$$

where $F$ is the corresponding $p \times p$ Fisher information matrix:

$F=\sum_{j=1}^{n} \frac{1}{\sigma^{2}\left(t_{j}\right)} \nabla_{\theta} h\left(t_{j}, \theta_{0}\right) \nabla_{\theta} h\left(t_{j}, \theta_{0}\right)^{T}$

and the symbol "." in (20) represents element-by-element vector multiplication and $\nabla_{\theta} h$ is the gradient that contains the derivatives of $h$ with respect to each component of the parameter vector $\theta$, that is the sensitivity of $h$ with respect to the parameters. Thus, GSF are defined only at the discrete time points $t_{1}, \ldots, t_{\mathrm{n}}$ where measurements are taken. Note that $g s\left(t_{k}\right)$ involves all the contributions of those measurements up to and including $t_{k^{*}}$. On the other hand the incremental GSF $\left(\mathrm{GSF}_{\text {inc }}\right)$, defined by:

$\operatorname{GSF}_{\text {inc }}\left(t_{k}\right)=F^{-1} \times \frac{\nabla_{\theta} h\left(t_{k}, \theta\right)}{\sigma^{2}\left(t_{k}\right)} \cdot \nabla_{\theta} h\left(t_{k}, \theta\right), \quad k=1, \ldots, n$

gives the information provided by the single measurements $t_{k}$. Note from (20) and (21) that the TSF have to be calculated in order to compute the GSF.

Remark The relationship between the GSF and the estimator of $\theta$ becomes clear if we introduce a continuous version of the parameter estimation problem supposing we have the measurements of the output for all $t \in[0, T]$. In this case the minimization of $J$ in (19) can be thought in a space of probability (continuous version) leading to:

$J(P, \theta)=\int_{0}^{T} \frac{y(t)-h(t, \theta)^{2}}{v^{2}(t)} d P(t)$

where $t_{\mathrm{i}} \in[0, T]$ and $P$ is a probability function defined in $[0, T]$. In this frame the Fisher information matrix becomes $F_{i j}(T, \theta)=\int_{0}^{T} \frac{1}{v^{2}(t)} \frac{\partial h(t, \theta)}{\partial \theta_{i}} \frac{\partial h(t, \theta)}{\partial \theta_{i}} d P(t)$ and its inverse is, asymptotically and approximately, the covariant matrix of the estimator $\hat{\theta}$ of $\theta$. The generalized sensitivity at $t \in[0, T]$ is the diagonal of $F\left(P, \theta_{0}\right)^{-1} F\left(P_{[0, t]}, \theta_{0}\right)$ (see Banks et al., 2008).

Now we can come back to the FP of EEG modeled by equations (1)-(5). In this case we cannot calculate the GSF corresponding to the PDE system that models the FP of EEG straightforward since instead of a dynamical system, in this case the independent variable is $x \in G \subset R^{3}$. The available data is now the value of the potential $u$ at given points on the scalp $x_{j}$ contaminated with noise and the observations are given by:

$y\left(x_{j}\right)=u\left(x_{j}, \theta\right)+e_{j}, \quad x_{j} \in \Gamma, \quad j=1, \ldots, n$

where the observation errors $e_{j} \in R$ are assumed to satisfy the standard statistical assumptions. We assume that there exists a value parameter $\theta_{0}$ such that the data set $\left\{y_{j}\right\}$ has the form given in (23) when $\theta=\theta_{0}$.
In Rubio et al. (2009) it was proved that choosing an arbitrary order for the observation points $x_{j}$, the function:

$\operatorname{GSF}_{\text {inc }}\left(x_{j}\right)=F^{-1} \times \frac{\nabla_{q} h\left(x_{j}, \theta\right)}{\sigma^{2}\left(x_{j}\right)} \cdot \nabla_{\theta} h\left(x_{j}, \theta\right), \quad j=1, \ldots, n$.

is well-defined, that is, the value of $\operatorname{GSF}_{\text {inc }}(x)$ at a given observation point $x \in \partial G$ does not depend on the order given to the observations points. Thus, we choose an order for the observation points on the scalp and perform the calculations.

\section{RESULTS}

We calculate the TSF and the GSF of $u$ with respect to $\sigma_{1}$ for a dipole source $J_{i}(x)=M \delta(x-q)$ for different locations $q \in G_{1}$ in a spherical head model. This approximation for the head allows us to calculate the solution by the series formula appearing in Zhang (1995). Differentiating this series with respect to $\sigma_{1}$ we obtain the sensitivity function $s_{1}$ for the case of nested homogeneous spherical sets. We compare their values to find out the information that they provide about the variations on the scalp potential $u$ when $\sigma_{1}$ changes. The values of the sensitivities were simulated considering that the observations are measurements of the electric potential on the scalp collected by means of a set of electrodes with 10-10B configuration and that the "spike-instants" where the values of $u$ are considered, were detected and marked on the data by experts. We consider the conductivity values in (2): $\sigma_{1}=0.33, \sigma_{2}=0.0042$, $\sigma_{3}=0.33(1 /(\Omega / m)$ ) (see Geddes and Baker, 1967). We choose an order to enumerate the electrode positions and calculate the GSF $F_{\text {inc }}$ and the TSF with respect to $\sigma_{1}$ for different radial and tangential dipoles. The results obtained are shown in the figures below. Since no comparison between sensitivities for different parameters is presented, we have not normalized the values and have plotted the values of the absolute value of TSF directly. In Figure 1 we plot the values of $\mathrm{GSF}_{\text {inc }}$ at the 20 electrode positions for the same dipole location $r=(0.3,0.4$, 0 ) and different dipoles moments, a radial one $M=(3,4,0)$ and a tangential one $M=(-4,3,0)$.

In Figure 2, we show the absolute value of the TSF on the scalp for the same dipole location $r_{q}=(0.3,0.4,0)$ and different dipoles moments. The stars indicate the positions of the scalp electrodes. In order to compare both sensitivity functions we have put an $\mathrm{M}$ at the position of the electrode where the two highest values of $\mathrm{GSF}_{\text {inc }}$ are achieved.

\section{DISCUSSION}

From the experiments we conclude that in this example TSF and GSF do not seem to provide the same information. It can be noted that in Figures 2B and D the maximum is not located at the electrode where TSF would suggest. Since the GSF is defined taking into account the influence that parameter variations have on the estimator $\hat{\theta}$, theoretically it seems to be more suitable to look at the values of the GSF before performing the parameter estimation. More examples in different domains had to be performed and a theoretical analysis about the information provided of both sensitivity functions must be done. In future, 

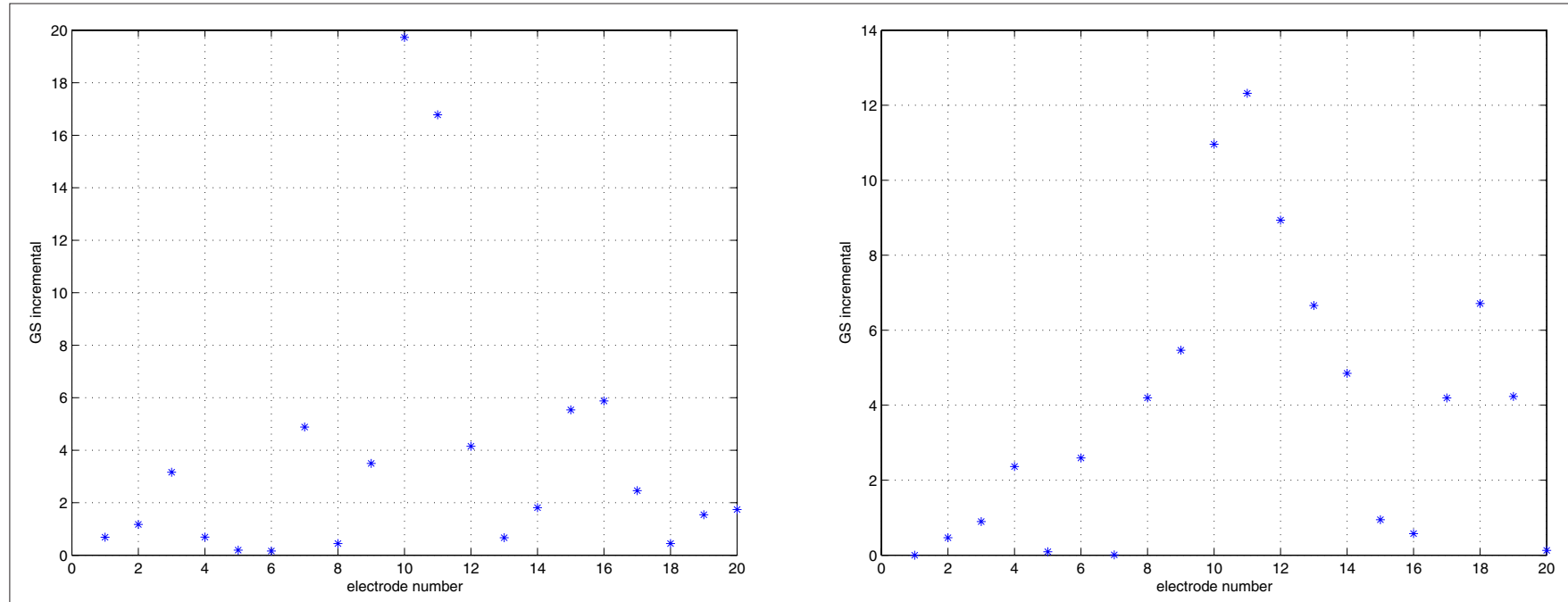

FIGURE 1 | Generalized sensitivity $\mathrm{GSF}_{\text {inc }}$ (in absolute value) for the dipole position $r_{q}=(0.3,0.4,0)$ and different moments: Right: $M=(3,4,0)($ radial) Left: $M=(-4,3,0)$.
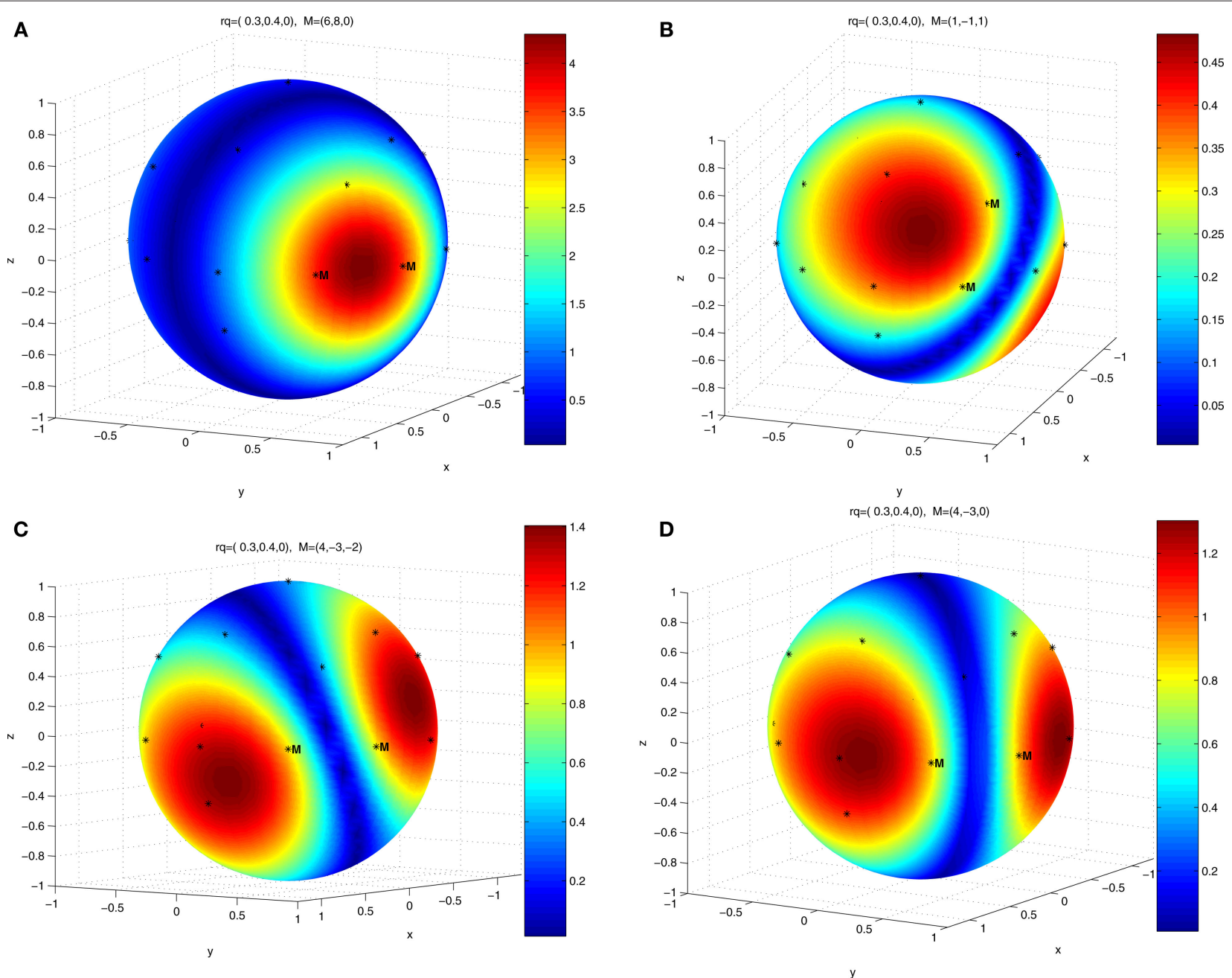

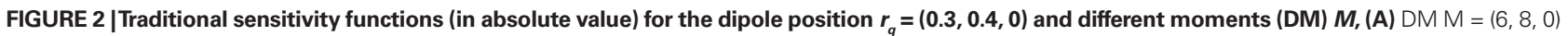
(radial) (B) DM M $=(1,-1,1)$ (C) $\mathrm{DM} \mathrm{M}=(4,3,-2)$ (D) DM M $=(4,-3,0)$ (tangential). 
based on these sensitivities, an optimal distribution of scalp electrodes may be determined in order to estimate the parameters of the model.

\section{REFERENCES}

Banks, H. T., and Bihari, K. L. (2001). Modeling and estimating uncertainty in parameter estimation. Inverse Probl. 17, 95-111.

Banks, H. T., Dediu, S., and Ernstberger. S. L. (2008). Sensitivity functions and their uses in inverse problems. $J$. Inverse III-Posed Probl. 15, 683-708, ISSN (Online) 1569-3945.

Burns, J.A., Lin, T., and Stanley, L. (2005). A Petrov-Galerkin finite element method for interface problems arising in sensitivity computations. Comput. Math. Appl. 49, 1889-1903.

El Badia, A. (2000). Inverse source problem in an anisotropic medium by boundary measurements. Inverse Probl. 16, 651-663.

Geddes, L. A., and Baker, L. E. (1967). The specific resistant of biological materials - a compendium of data for the biomedical engineering and physiologist. Med. Biol. Eng. 5, 271-293.

Hamalainen, M., Hari, R., Ilmoniemi, R. J., Knuutila, J., and Lounasmaa, O. (1993). Magnetoencephalography, theory, instrumentation and applications to noninvasive studies of the working human brain. Rev. Mod. Phys. $65,414-487$.

Kappel,F., and Batzel,J. (2006). “Sensitivity analysis of a model of the cardiovascular system," in Proceedings of the 28th IEEE EMBS Annual International Conference, New York.

Rubio, D., Troparevsky, M. I., and Saintier, N. (2009). "Generalized sensitivity analysis for an elliptic equation with interfaces," in Proceedings of XIII Reunión de trabajo en procesamiento de la Información y control, RPIC 09, Rosario, Argentina.

Sarvas, J. (1987). Basic mathematical and electromagnetic concepts of the

\section{ACKNOWLEDGEMENTS}

This research was supported in part by the Air Force Office of Scientific Research under grant FA9550-10-1-0037

biomagnetic inverse problem. Phys. Med. Biol. 32, 11-22.

Stanley, L. G. (1999). Computational Methods for Sensitivity Analysis with Applications to Elliptic Boundary Value Problems. Ph.D. thesis, Department of Mathematics, Virginia Tech, Blacksburg, VA.

Thomaseth, K., and Cobelli, C. (1999) Generalized sensitivity functions in physiological system identification. Ann. Biomed. Eng. 27, 607-616.

Troparevsky, M. I., and Rubio, D. (2003). On the weak solutions of the forward problem in EEG. J. Appl. Math. 12, 647-656.

Zhang, Z. (1995). A fast method to compute surface potentials generated by dipoles within multilayer anisotropic spheres. Phys. Med. Biol. 40, 335-349.

Conflict of Interest Statement: The authors declare that the research was conducted in the absence of any commercial or financial relationships that could be constructed as a potential conflict of interest.

Received: 04 February 2010; paper pending published: 29 June 2010; accepted: 30 August 2010; published online: 30 September 2010.

Citation: Troparevsky MI, Rubio D and Saintier N (2010) Sensitivity analysis for the EEG forward problem. Front. Comput. Neurosci. 4:138. doi: 10.3389/ fncom. 2010.00138

Copyright (C) 2010 Troparevsky, Rubio and Saintier. This is an open-access article subject to an exclusive license agreement between the authors and the Frontiers Research Foundation, which permits unrestricted use, distribution, and reproduction in any medium, provided the original authors and source are credited. 